\title{
Las «benefit corporations» norteamericanas
}

\author{
Iñigo Zavala Ortiz de la Torre \\ Universidad de Deusto
}

Sumario: I. Introducción. II. Concepto. III. Fundamento. IV. Marco legal existente antes de la aprobación de las Benefit Corporations. V. Régimen Jurídico. VI. A modo de conclusión. VII. Bibliografía.

Resumen: La Benefit Corporation es la nueva forma de empresa social surgida en el panorama legislativo norteamericano. Sobre la base de una estructura de sociedad mercantil capitalista, se pretende poder obtener resultados en beneficio del interés general. Este tipo social, trata de aunar los legítimos intereses financieros de los accionistas, con los de los otros stakeholders de la compañía. Para ello los administradores deberán aplicarse en la obtención de beneficios, pero a través del ejercicio de una actividad empresarial que, en su conjunto, tenga un impacto positivo en la sociedad y en el medio ambiente. Este impacto positivo, deberá poder ser, a su vez, objeto de evaluación a través de su contraste con normas estandarizadas, ideadas y aprobadas por terceros.

Palabras clave: Interés general público, stakeholders, intereses específicos, empresa social, benefit corporation, evaluación por un tercero independiente, impacto social, impacto medio ambiental.

Abstract: The Benefit Corporation is a new type of social enterprise that has been enacted in the United States legislation. With the basis of a capitalist corporate legal entity, this corporation should pursue a specific public benefit. The benefit corporation tries to encompass the legitimate financial rights of the shareholders with those of the rest of the stakeholders of the company. So the Directors should work to generate returns for the shareholders, but through the exercise of an activity that should has a material positive impact on society and the environment, taken as a hole. This positive impact should be assessed against a third-party standard.

Key words: General public benefit, stakeholders, specific public benefit, social enterprise, benefit corporation, third party standard, social impact, environmental impact. 


\section{Introducción}

Una economía de mercado, por definición, no es capaz de estimular la producción de ciertos bienes y servicios que por tener como destinatarios, o mejor dicho, por ser sus beneficiarios el público o la sociedad en general, no generan en sus fabricantes ganancia directa alguna. Se espera, por tanto, que sea el Estado el que supla estas carencias, ayudando además a reducir las diferencias que la limitación en el acceso a estos bienes, se producen entre individuos y regiones. Tradicionalmente, en los Estados Unidos$^{1}$, al igual que en el resto del mundo desarrollado, han existido organizaciones privadas, que bajo la denominación de «non-profit» (o sin ánimo de lucro) han tratado de ayudar a los damnificados por los fallos del mercado (y no solo del Mercado²), allí donde el Estado no llegaba.

Desde el inicio de la década de los años 80 un conjunto de «empresarios sociales ${ }^{3} »$ norteamericanos empezó a cuestionarse las formas legales habilitadas por la ley hasta ese momento, para la consecución de determinados fines sociales y medio ambientales. En efecto, si bien para el logro de determinados objetivos las formulas tradicionales ${ }^{4}$ eran

1 Katz, R.A. y Page, A., "The Role of Social Enterprise», Vermont Law Review, vol. 35 de 2010, p. 60. En este artículo se realiza además una clarificadora clasificación de las distintas clases de «non-profit enterprises».

2 Benedicto XVI, Carta Encíclica Caritas in Veritae de 7 de julio de 2009, Ed. San Pablo, Madrid, 2009, Cap. III, Epígrafe 34, p. 25. En donde se afirma: «Hace tiempo que la economía forma parte del conjunto de los ámbitos en que se manifiestan los efectos perniciosos del pecado. Nuestros días nos ofrecen una prueba evidente. Creerse autosuficiente y capaz de eliminar por sí mismo el mal de la historia ha inducido al hombre a confundir la felicidad y la salvación con formas inmanentes de bienestar material y de actuación social. Además, la exigencia de la economía de ser autónoma, de no estar sujeta a «injerencias» de carácter moral, ha llevado al hombre a abusar de los instrumentos económicos incluso de manera destructiva. Con el pasar del tiempo, estas posturas han desembocado en sistemas económicos, sociales y políticos que han tiranizado la libertad de la persona y de los organismos sociales y que, precisamente por eso, no han sido capaces de asegurar la justicia que prometían». Ver también en: http://www. conferenciaepiscopal.es/documentos/benedictoXVI/enciclica/CaritasVeritate.pdf. Última entrada 27 de mayo de 2013.

3 Katz, R.A. y Page, A., «The Role of Social...», op.cit., p. 60. Para estos autores, profesores de la Universidad de Indiana, el Social Entrepreneur es «An ambitious person who seeks social change on a large scale, characteristically through earned income strategies». Así mismo The New York Times, comparó a estos empresarios con los líderes del movimiento a favor de los derechos civiles. «The age of ambition», NYT, 28 de enero de 2008.

4 Katz, R.A. y Page, A., "The Role of Social...», op.cit. p. 31. En la que se define la for-profit social Enterprise como «la organización que, a diferencia de las sin ánimo de lucro, no tienen limitación a la hora de repartir beneficios, y que a diferencia de una con ánimo de lucro, no está limitada por una norma que le obliga a maximizar en exclusiva el interés de los accionistas». 
apropiadas, sin embargo, para otros muchos eran estructuras demasiado rígidas, ancladas en la concepción de la caridad de hace más de cien años, y que por lo tanto no se ajustaban a las nuevas inquietudes del inicio del siglo $x x^{5}$. Y así, tal y como afirma Kelley " ${ }^{6}$ Social entrepreneurs as a group are shifting their focus away from the non-profit sector, traditionally in charity and philanthropic activities, towards entrepreneurial private-sector oriented business activities». Fruto de estas inquietudes surgieron, entre otras7, las Low Profit Limited Liability Company ${ }^{8}$ (ó L3C), y la Californiana Flexible Purpose Corporation ${ }^{9}$ (FPC), en ambos casos sociedades capitalistas con ánimo de lucro, pero en las que el fin social es la persecución del interés general, antes que la obtención de beneficios. El problema de ambas (y por lo que no tuvieron la aceptación esperada, al resultar poco atractivas incluso para inversores con gran sensibilidad social) fue la denominada asset lock o nondistribution constrain. Es decir una estructura demasiado rígida que impide la distribución de todos los beneficios.

En 2008, de nuevo el Congreso de California, aprobó una modificación del La Ley de Sociedades Californiana, que autorizaba a los Administradores a tomar en consideración motivaciones medio ambientales a la hora de adoptar decisiones de gestión. Nunca entró en vigor debido a que las «imprevisibles consecuencias» que tal medida podría

5 Tenemos que recordar que en Europa la legislación existente a este respecto, además de mucho más avanzada y arraigada socialmente, ofrece una variedad de sociedades (Sociedades Laborales, y Sociedades Cooperativas de toda clase) que, o no existen en los Estados Unidos, o tienen una aplicación práctica escasa.

6 Kelley, T., "Law and Choice of Entity on the Social Enterprise Frontier», Tulane Law Review. vol. 84 de 2009, p. 337.

7 Otra forma híbrida, aprobada en el Estado de Wisconsin en el año 2001, es la Limited Cooperative Association (LCA). Con el objeto de solventar los problemas de acceso al capital privado, que tradicionalmente se ha atribuido a las S. Cooperativas en EE.UU., permite la remuneración y el derecho al voto de aportaciones puramente financieras al capital social. No obstante este tipo social ha sido duramente criticado por entender parte de la doctrina que se alejaba de los principios cooperativos. Además ha tenido escaso éxito, habiéndose contabilizado hasta 2008 solo 46 LCA. Ver en University of Wisconsin Center for Cooperatives. Staff Paper N. ${ }^{\circ}$ 7, 2008, en http://www.uwcc. wisc.edu/pdf/Staff\%20Papers/Staff\%20Paper\%207.pdf Última entrada 2 de julio de 2013.

8 Ver en http://www.dmlp.org/legal-guide/low-profit-limited-liability-company. Última entrada 31 de mayo 2013.

9 Ver en http://leginfo.legislature.ca.gov/faces/billNavClient.xhtml?bill_ $\mathrm{id}=201120120$ SB201. Para ver una comparativa entre la FPC y la Benefit Corporation ver http://www.hansonbridgett.com/Publications/articles/2012-09-flexible-purpose.aspx. Última entrada 31 de mayo de 2013. 
deparar, hicieron que el Gobernador Arnlod Schwarzenegger la vetase $^{10}$.

A pesar de todas las iniciativas previamente relacionadas, ninguna de ellas fue capaz de obtener la aprobación conjunta de los empresarios, los inversores y los consumidores. Con el objetivo de dar solución a esta discordia B Lab ${ }^{11}$ propuso la «Benefit Corporation».

Esta nueva forma social no pudo ser mejor acogida por la doctrina norteamericana. Y así, y de entre las diferentes clases de Social Enterprise $^{12}$ se afirma que "The most ambitious of these new business forms is the Benefit Corporation» y también "The Benefit Corporation is perhaps the most ascendant Social Enterprise innovation today ${ }^{13} »$. No obstante, también han existido críticas $^{14}$. Desde los que la consideran

10 Ver en ftp://leginfo.public.ca.gov/pub/07-08/bill/asm/ab_2901-2950/ab_2944_ vt_20080930.html. Última entrada 28 de mayo de 2013. En este texto en el que el gobernador justifica su veto, se expone que «la protección del accionista ha sido vital para California». Luego a sensu contrario nos dice que la maximización del valor de la acción en beneficio solo de los accionistas ha de seguir siendo el único referente de los Administradores.

11 B. Lab. es una asociación sin ánimo de lucro de gran prestigio e influencia en los Estados Unidos. Tal y como se afirma en su página web, B Lab es una organización que apoya y sirve, a un movimiento global de empresarios que utilizan sus negocios y empresas para resolver problemas sociales y medio ambientales. B Lab realiza su labor a través de los siguientes tres tipos de iniciativas interrelacionadas: a) la denominada «Certificación B.Corp» que se otorga a aquellas sociedades que lo solicitan y cumplen determinados estándares sobre compromisos sociales; b) lo que ellos denominan «Passing legislation» ó labor de lobby en los congresos de los estados federados. Se trata de «vender» o «convencer» a los congresistas para que aprueben las propuestas de Benefit Corporations que esta organización presenta. Además tratan de persuadirlos para que utilicen el modelo por ellos ideado (lo han conseguido casi, siempre; y si no con muy pocos cambios). Por ejemplo en el caso del Estado de Delaware, ha sido ella misma la encargada - como Key Supporter - de presentarla ante su cámara legislativa. c) a través del Global Impact Investing Rating System (GIIR), mediante el cual se buscan y evalúan empresas socialmente responsables, al objeto de que inversores y fondos que hayan decidido invertir en este tipo de empresas, tengan acceso a las mismas.

12 Para la "Social Enterprise Alliance», una Social Enterprise o Empresa Social, es: "Social Enterprises are businesses whose primary purpose is the common good. They use methods and disciplines of business and the power of the marketplace to advance their social, environmental and human justice agendas». Ver en https://www.se-alliance. org/why\#whatsasocialenterprise. Última entrada 3 de julio de 2013.

13 Munch, S., «Improving the Benefit Corporation:How Traditional Governance Mechanisms can Enhance the innovative new Business Form». Northwestern Journal of Law and Social Politics, vol. 7 de 2012. p. 170.

14 Ver artículos de Celia Taylor del 20 de febrero de 2013, titulados «Benefit Corporations Expand but not with out Criticism» en http://www.theracetothebottom. org/home/benefit-corporations-expand-but-not-without-criticism-part-1.html. (Parte 1) y http://www.theracetothebottom.org/home/benefit-corporations-expand-but-notwithout-criticism-part-t.html. (Parte 2). Última entarda 2 de julio de 2013. 
«superflua» por entender que sus objetivos pueden ser cumplidos por la legislación actualmente en vigor ${ }^{15}$ hasta los que la consideran demasiado «conservadora ${ }^{16}$ ».

También los legisladores, y la sociedad civil en general, han sido tremendamente receptivos. Desde el inicio del año 2010 han sido 15 los Estados ${ }^{17}$ norteamericanos los que ya han aprobado, -y 14 los que están en trámite ${ }^{18}$ - las reformas legislativas encaminadas a la creación de este nuevo tipo social. Entendemos que tanto su novedad ${ }^{19}$, como su rápida acogida en los estados más importantes del aquél país, justifican estas líneas que, además, son un lógico cierre a nuestro anterior trabajo publicado en esta misma revista ${ }^{20}$.

A efectos didácticos utilizaremos como patrón sobre el que efectuar la presente exposición, tanto el Libro Blanco como el Model Bene-

15 Noket, N., Ver en «The Harvard Law School Forum on Corporate Governance and Financial Regulation»: http://blogs.law.harvard.edu/corpgov/2012/05/13/benefitcorporations-vs-regular-corporations-a-harmful-dichotomy/. Última entrada 28 de mayo de 2013.

16 Ver en «New Economy Working Group»: http://www.neweconomyworkinggroup.org/search/node/benefit\%20corporation. Última entrada 30 de mayo de 2013.

17 En los Estados Unidos, la legislación de Sociedades está reservada a los Estados Federados. En efecto, siguiendo la «internal affairs doctrine» las cuestiones societarias de la compañía serán reguladas por la Ley del Estado en la que los socios hayan decidido inscribir la sociedad. No es necesario, además que la sociedad tenga algún tipo de vinculo con el Estado en el que se decide inscribir («Incorporate») la Sociedad. Sólo aquellas materias que por su trascendencia y posible impacto frente a terceros, como es la emisión de Valores (Securities Act de 1933) la regulación del Mercado de Valores (Securities Exchange Act de 1934) son de competencia Federal. Ver Bainbridge, S. M., Corporate Law, Ed. Thomson/West. 2. ${ }^{a}$ edición, 2009, p. 8.

18 Los que han aprobado ya esta legislación son: Arizona, si bien no entrará en vigor hasta el día 31 de diciembre de 2014 (la ley fue aprobada muy recientemente, en concreto el 30 de abril de 2013), Arkansas aún no en vigor, California, Colorado entrará en vigor el 1 de abril de 2014 (la ley fue aprobada el día 1 de mayo de 2013), Hawaii, Illinois, Maryland, Massachusetts, Louisiana, New Jersey, New York, Pennsylvania, Vermont, Virginia, y Washington D.C. En estudio Alabama, Connecticut, Delaware, Florida, lowa, Montana, Nevada, North Carolina, Oregon, Rhode Island, Texas, y West Virginia.

19 Son ya 182 las compañías listadas en la página web oficial de la Benefit Corporations, si bien no están incluidas todas las que han adoptado esta forma social. Ver en http://benefitcorp.net/find-a-benefit-corp/search. Entre ellas esta la compañía de ropa deportiva Patagon, con sede en California, y Blessed Coffee que «devuelve la mitad de sus beneficios a la cooperativa etíope que es su principal proveedor, para que esta lo invierta a su vez en escuelas, hospitales o en cualquier otra mejora de la comunidad en la que tiene su sede». Última entrada 27 de mayo de 2013.

20 Zavala Ortiz de la Torre, Iñigo, "La pugna entre el shareholder primacy model y la stakeholder theory en la doctrina y práctica anglosajona. Estado de la cuestión», Deusto Estudios Cooperativos, 2, 2013, pp. 103-132. 
fit Corporation Legislation ó $M B C L^{21}$, ideados y redactados por B Lab. Este último Model es la propuesta realizada por la citada organización, sobre cuál debería ser la formula a adoptar por los distintos Estados a la hora de incorporar a su legislación esta nueva forma social. Para ello, en el apartado IV del presente trabajo, y tras la exposición de cada una de las secciones en las que está dividida la $\mathrm{MBCL}$, realizaremos un breve comentario que tiene como única finalidad el facilitar la mejor comprensión de este nuevo tipo societario.

La estructura del presente trabajo, se articula de la siguiente forma: Un apartado II en el que trataremos de ofrecer al lector el concepto descriptivo de lo que es una Benefit Corporation; un apartado III en el que nos haremos eco de la fundamentación teórica sobre la que los proponentes cimentan la necesidad de este nuevo tipo social; un apartado IV en el que analizaremos el marco legal existente en estos momentos en los EE.UU. (para comprender mejor la necesidad de la Benefit Corporation en aquél país); un apartado $V$ en el que pasamos a trasponer la esencia del régimen jurídico propuesto para este nuevo tipo social; y Conclusiones en el apartado VI.

\section{Concepto}

Tanto el White Paper sobre las Benefit Corporations, (que tiene como sub-título «La necesidad y razón de las Benefit Corporations: El porqué es el tipo social que mejor se adecua a las necesidades de los empresarios sociales, de los inversores y del público en general»), como el Model Benefit Corporation Legislation nos dan la misma definición (bastante lacónica) de este nuevo tipo social. En el art. 102 de este último, simplemente se determina que la Benefit Corporation, es una sociedad capitalista ${ }^{22}$ que: «(1) ha elegido estar regulada por las disposiciones de este capítulo y (2) cuyo estatus como Benefit Corporation sigue vigente». El White Paper, resalta las siguientes características como las más sobresalientes del nuevo tipo social: 1) Su objeto social es el crear un impacto real y positivo en la sociedad; 2) Una ampliación de los deberes de los administradores que tendrán que tener en cuenta, - a la hora de tomar sus decisiones-, no solo los intereses fi-

21 Verlos en http://benefitcorp.net/for-attorneys/benefit-corp-white-paper. En el presente trabajo, hemos trabajado sobre la versión de fecha 18 de enero de 2013. Última entrada 31 de mayo de 2013.

22 Traducimos el término «Business Corporation» como «Sociedad Capitalista», sin distinguir entre Sociedad Anónima y Sociedad de Responsabilidad Limitada. 
nancieros de los accionistas, sino también los de otros «Stakeholders» (ya veremos más adelante a quienes otorga el $\mathrm{MBCL}$ esta cualidad); 3) La obligación de informar anualmente sobre la gestión social y medio ambiental, así como la evaluación de la misma, utilizando para ello unos estándares, desarrollados por una tercera organización, que sean comprensibles, creíbles, independientes y transparentes.

Es decir, una Benefit Corporation es igual que una sociedad capitalista «normal» en todos sus aspectos, excepto en uno: está legalmente obligada a perseguir el interés general ó interés público. Las Benefit Corporations son Sociedades capitalistas a las que no falta el ánimo de lucro. Pero son Sociedades en las que su ánimo de lucro ha sido redefinido. Como cualquier otra Sociedad capitalista, una Benefit Corporation tiene que tratar de obtener un beneficio para sus accionistas; pero el camino a través del cual ese beneficio debe ser obtenido, es mediante la gestión de la compañía de forma respetuosa con el medio ambiente y con el interés general de la sociedad ${ }^{23}$. Para ello, deberán sus administradores tener en cuenta los intereses de los colectivos directamente relacionados con la Sociedad (también denominados Stakeholders), es decir los accionistas, los trabajadores, los clientes, los proveedores, los acreedores, la comunidad en donde la empresa se ubica, y el medio ambiente ${ }^{24}$.

La Benefit Corporation con sus beneficios podrá, bien repartirlos vía dividendos entre sus accionistas, bien retenerlos dotando para ello las reservas legales y estatutarias. Exactamente el mismo régimen que cualquier otra Sociedad capitalista y estando sometida a las mismas obligaciones contables, fiscales y laborales. Realmente las Benefit Corporations han sido una reacción a la predominante (aunque cada vez más cuestionada) shareholder primacy ${ }^{25}$, tal y como tendremos ocasión de comentar con posterioridad.

No es fácil encontrar la traducción del término inglés a nuestra lengua. Pero exclusivamente con la idea de dar al lector una visión aproximada, y combinando las denominaciones de tipos sociales de similares características y finalidades, aprobados recientemente en California, la «Flexible Purpose Corporation» (o Sociedad de finalidades flexibles); en

${ }^{23}$ A los efectos de evitar posibles en este trabajo, cuando nos queramos referir a la «Sociedad» como forma asociativa, objeto de regulación por nuestro derecho mercantil, la escribiremos con «S» mayúscula; y cuando queramos referirnos a la «sociedad» civil, como concepto de la ciencia social, lo haremos con «s» minúscula.

24 Ver art. 301 en la página 14 de este trabajo.

25 Stout, L., The Shareholder Value Myth, Ed. Berret-Koehlet Publishers. Inc., San Francisco, 2012. 
El Reino Unido, las «Community Interest Company» ó Sociedad en Interés de la Comunidad; y en Bélgica, las "Société à Finalité Sociale» ó Sociedad con Finalidad social26, quizás pudiéramos proponer la de "Sociedad de Capital con Finalidad Social». La cual preferimos a la de Sociedad de Capital de Interés Público, por entender que esta, es más equívoca.

\section{Fundamento}

Los proponentes de este nuevo tipo social, han fundamentado su necesidad, y racionalidad, desde tres puntos de vista distintos.

- Desde el de los consumidores. Una creciente parte de la población norteamericana trata de adecuar sus hábitos consumistas a sus «valores morales». Aproximadamente 68 millones de consumidores de aquél país han manifestado su deseo de que sus «compras» sean compatibles o coherentes con su sentido de la responsabilidad social y medio ambiental. Existen estudios que han constatado que el $49 \%$ de los Americanos boicotearían a compañías cuya actuación fuese contraria, o incluso no fuera dirigida a promover el mejor interés de la sociedad en su conjunto ${ }^{27}$. Así mismo, más recientes publicaciones han concluido que, para el caso de equivalencia de precio y calidad, el $86 \%$ de los consumidores encuestados cambiarían sus actuales marcas por otras que fueran socialmente más responsables ${ }^{28}$.

Pero también es cierto, que en determinada medida la confianza del consumidor en productos etiquetados como «verdes», "sostenibles», ó «responsables con el medio ambiente» se está deteriorando. Ello es debido al abuso que de estos términos hacen los responsables de marketing de las grandes empresas. Además es muy difícil el calibrar hasta que punto tales afirmaciones son verdaderas, al no existir unos estándares homogéneos contra los que contrastarlos. Este problema - que ha sido denominado como "greenwashing»— ha frustrado las expectativas de muchos consumidores. En efecto, hasta ahora, las certificaciones

26 Munch, S., «Improving the Benefit...», op. cit, p. 184.

27 Bonini, S., McKillop, K., y Mendonca, L., «The trust gap between consumers and corporations» en The McKinsey Quarterly, vol. 10. 2007. En «White Paper. The need and rationale...», op. cit.

28 Cone Comunications, 2010. Cone Cause Evolution \& Enviroment Survey, Cone Research Report, 8 (2007). Ver en http://www.coneinc.com/files/2007ConeSurveyReport. pdf. Última entrada 20 de junio de 2013. 
y estándares que existen, tienen un ámbito de aplicación demasiado estrecho (acreditan los orígenes o modos de fabricación de productos concretos, o incluso determinadas filosofías que alientan la prestación de variados servicios). Es por ello necesario el desarrollo de normas estandarizadas que provean de una visión general de la compañía, en lo que se refiere a su gestión social y medio ambiental, así como una evaluación de sus logros a este respecto, clara, contrastable y verificable.

- Desde el de los Inversores. Los Inversores Socialmente Responsables (o SRI en el acrónimo inglés de Socially Responsible Investing) han crecido, en los 30 últimos años de forma exponencial. Representan en estos momentos el $10 \%$ de los activos gestionados por Inversores Institucionales y entidades financieras. En efecto, son cada vez más los fondos, las sociedades de capital riesgo, sociedades de inversión y particulares que buscan dirigir sus inversiones hacia empresas socialmente responsables.

Al igual que los consumidores, los inversores, carecen de las herramientas necesarias para detectar y evaluar, si la autoproclamada conciencia social o medio ambiental de muchas Sociedades es cierta o nos encontramos, como antes hemos comentado, ante una mera estrategia de marketing o "greenwashing». De hecho, en los mercados financieros, se está demandando cada vez con mayor intensidad el que las Sociedades emitan un informe sobre sostenibilidad, como documento independiente a sus Cuentas Anuales ${ }^{29}$.

- Desde el de los Empresarios. Los empresarios con inquietudes sociales han ido ganando visibilidad en el paisaje del mundo de los negocios. Probablemente el espaldarazo definitivo y reconocimiento económico-social de su actividad fue la concesión, en 2006, del Premio Nobel de la Paz a Muhammad Yunus, por sus pioneros trabajos sobre microfinanzas, en concreto en el Banco Grameen de Bangladesh.

Esta «casta» de empresarios, critica que en los Estados Unidos, el régimen jurídico de las Sociedades capitalistas esté concebido de forma que lo que se garantiza es la obtención de cuantos más beneficios mejor, sin margen alguno para la responsabilidad social.

${ }_{29}$ Ver en http://www.issgovernance.com/docs/2011ESGPreview. Institutional Shareholder Service. (ISS) Corporate Social Issues: A 2011 Proxy season Preview: United States 1 (2011), en donde literalmente se dice «Institutional Investors appear to be increasingly incorporating social and environmental considerations into their proxy voting decisions as demonstrated by voting trends and institutional investors initiatives». 
La suma de las preocupaciones de estos tres distintos sectores de la sociedad, así como las limitaciones del actual marco legal, hicieron que la propuesta liderada por B Lab, haya tenido una aceptación realmente inesperada.

\section{Marco legal existente antes de la aprobación de las benefits corporations}

En este apartado estudiaremos cual era el marco legal existente en la mayoría de los Estados norteamericanos. Si bien realizaremos un breve resumen de la situación, para un conocimiento más profundo de esa realidad, nos remitimos a nuestro anterior artículo, publicado en el número 2 de esta revista ${ }^{30}$.

Como en el mismo señalábamos, existen 40 estados que han aprobado los llamados «constituencies statutes» que son leyes, en las que se permite que el órgano de administración pueda tomar en consideración, a la hora de tomar sus decisiones, no solo el interés de los accionistas, sino también el de otros interesados o stakeholders. Esto significa, que los accionistas no podrían demandar a los administradores por adoptar decisiones que no tuvieran como objetivo directo incrementar las ganancias, o el valor de la acción en el exclusivo beneficio de los accionistas.

Por el contrario, los non-constituencies statutes prohíben expresamente a los administradores de las Sociedades, el tomar en consideración intereses de stakeholders distintos de los accionistas. Entre ellos se encuentra el estado de Delaware ${ }^{31}$ en el que tienen su domicilio 900.000 compañías y más del $50 \%$ de las sociedades cotizadas de los Estados Unidos 32 .

30 Zavala Ortiz de la Torre, Iñigo, «La pugna entre el shareholder primacy model», op. cit.

31 Sin embargo incluso en el Estado de Delaware se encuentra en trámite de aprobación las Benefit Corporations ver en Forbes Magazine: http://www.forbes.com/sites/ annefield/2013/04/25/what-will-be-the-first-delaware-benefit-corporation/. Recordar que la legislación de este Estado, es la quinta esencia del liberal capitalismo más descarnado, con ninguna cesión a los intereses de colectivos distintos al de la propia dirección de la compañía, y los accionistas. Última entrada 2 de julio de 2013.

32 La legislación societaria de este Estado, en donde tienen su domicilio social la mayoría de las grandes empresas norteamericanas, se caracteriza por la permisividad de sus disposiciones legales, lo cual deja un amplio margen a la autonomía de la voluntad de los socios. Esta permisibilidad en algunos de los Estados Federados ha dado lugar a la denominada «race to the bottom» o carrera hacia al abismo. Término con el que la doctrina señala la carrera hacia la permisibilidad en la que algunos Estados de la Unión están in- 
Aunque existen autores ${ }^{33}$ que mantienen que con los constituencies statutes es posible que la Sociedad pueda perseguir fines sociales - y por lo tanto apartarse del principio de que solo existe para maximizar beneficios-, la práctica nos muestra que la realidad es bien distinta. En efecto, la falta de jurisprudencia aplicable (ya que de hecho casi no han existido demandas en las que se solicitaba la condena de los administradores por haber tenido en cuenta los intereses de otros stakeholders distintos de los accionistas); la falta de mecanismos para que los stakeholders puedan hacer valer sus intereses 34 ; así como el contexto en el que esta legislación fue aprobada35, hace que los administradores de estas sociedades, sean muy cautos a la hora de acometer objetivos sociales. En resumen, la inseguridad jurídica reinante en este aspecto disuade a muchos administradores y accionistas de utilizar el vehículo de las sociedades capitalistas para la consecución de fines sociales y medio ambientales.

Por lo tanto nos encontramos, de hecho, con que la teoría prevalente en este ámbito es la llamada Shareholder Primacy, o teoría que antepone los intereses de los accionistas frente a los cualquier otro Stakeholder.

\section{Regimen jurídico}

Ante esta situación, un grupo de empresarios con inquietudes sociales, se organizo entorno a la organización sin ánimo de lucro ${ }^{36}$ B Lab antes comentada. Esta organización, además de encargar a prestigiosos profesores y despachos de abogados la redacción de lo que pudiera ser la legislación básica de un nuevo tipo social —que compaginase los atractivos de la sociedad capitalista y los de una sociedad con objetivos sociales y medio ambientales-; desarrollo una intensa labor de lobby

mersos, con el objeto de atraer a su jurisdicción al mayor número de compañías. El objetivo perseguido es el de que las tasas e impuestos que estas deben pagar por domiciliarse en cada Estado, nutran de recursos las arcas del estado en cuestión. Ver Romano, R., The Genius of American Corporate Law, Ed. The AEl Press, Washigton DC, 1993.

33 Mitchel, L., "A theoretical and Practical Framework for Enforcing Corporate Constituency Statutes», Texas Law Review, vol. 70, 1992, p. 579.

34 Munch, S., «Improving the Benefit Corporation», op. cit., p. 181, en donde afirma que «incluso en Idaho y Arizona en donde los Constituency Statutes, exigen a los Administradores considerar tanto el corto como el largo plazo, en el contexto de una OPA, la legislación de estos Estados, no regula los mecanismos coercitivos necesarios para que se pueda exigir a los Administradores, que efectivamente así lo hagan».

35 Keay, A., The Corporate Objetive, Ed. Edward Elgar Publishing Limited, Massachusetts, 2011, p. 58.

36 Una organización sin ánimo de lucro puede ser el equivalente a una Asociación en la legislación Española. 
ante los líderes de los dos partidos norteamericanos. Esta actuación, a nivel de cada Estado Federado, sin duda ha tenido un rotundo éxito dado los resultados hasta hoy alcanzados y comentados en la Introducción.

El proyecto fue concebido de forma que fuera fácilmente incardinable en las vigentes leyes o códigos ${ }^{37}$ de Sociedades en cada uno de los Estados. La idea era la introducción de un nuevo capítulo en las leyes vigentes. Este nuevo capítulo regularía en exclusiva el régimen jurídico de este nuevo tipo social. De esta forma, y según manifiestan sus autores $^{38}$, se evitaría el tener que adecuar la legislación de la Benefit Corporation cada vez que se produjera una modificación del régimen legal de las Sociedades en cada Estado. Lo cierto es que las modificaciones sancionadas hasta el momento, así como los proyectos en trámite de aprobación legislativa, son muy semejantes. Todos ellos siguen fielmente el «Model Benefit Corporation Legislation» antes mencionado; afirmando por lo tanto, que el estudio de este es suficiente para poder realizar una exposición comprensiva de la novedad legislativa.

El resultado es el siguiente:

\section{CAPITULO-_39. THE BENEFIT CORPORATION}

Sección I. Disposiciones Preliminares

$\mathrm{Art}^{40}$. 101.Aplicación y efectos del presente Capítulo

A) Adopción de la cualidad de Benefit Corporation

Esta cualidad, se puede obtener, bien en el momento de la constitución de la Sociedad, o bien con posterioridad, cuando una compañía ya existente decide adoptar esta nueva forma social.

37 Nos referimos a sociedades mercantiles capitalistas, es decir las equivalentes a nuestras Sociedades Anónimas y de Responsabilidad Limitada. Para una más completa relación de los actuales tipos de sociedades mercantiles capitalistas, tanto con responsabilidad limitada como ilimitada, norteamericanas y españolas, ver Guerra Martín, G., El Gobierno de las Sociedades Cotizadas Estadounidenses: Su influencia en el movimiento de reforma del Derecho Europeo, Ed. Aranzadi, Cizur Menor, 2003, p. 68.

38 "White Paper. The need and rationale for the Benefit Corporation...», op. cit., p. 15.

39 Tal y como hemos comentado lo que se pretende es la introducción de un nuevo capítulo en el lugar en el que, a juicio de los legisladores, mejor encaje tenga teniendo en consideración la estructura de cada Ley de Sociedades. Por ello, la numeración que hemos dado a los artículos en el presente trabajo es aleatoria, y a los solos efectos de su exposición ordenada.

40 En este trabajo utilizaremos en término «artículo», en vez del original «section», por entender que facilita la comprensión del mismo. 
En el primer caso, y tal y como dispone el Art. 103 del MBCL, simplemente es necesario el que se haga constar expresamente en los estatutos (articles of incorporation) que se trata de una Benefit Corporation. En la denominación social, por lo tanto, no tiene que hacerse constar esta circunstancia ${ }^{41}$.

En el segundo caso, en el Art. 104, se dispone que una Sociedad capitalista, válidamente constituida, puede convertirse en una Benefit Corporation, a través de la modificación de sus estatutos sociales, de forma que en estos, se haga expresa mención a que la Sociedad es una Benefit Corporation. En cuanto a la mayoría necesaria para adoptar este acuerdo, la MBCL se remite a la exigida por la Ley de cada estado para realizar una modificación de estatutos ${ }^{42}$. De todas formas la propuesta de la MBCL (Ver definición de "Minimun Status Vote» en el Art. $\left.102^{43}(a)\right)$, es de una mayoría de dos tercios. Propuesta seguida por casi todos los Estados.

Finalmente, el último Art. de esta Secc., el 105, regula cuando una Benefit Corporation puede dejar de serlo voluntariamente. A estos efectos será necesaria una modificación de estatutos en la que se haga desaparecer la mención requerida para serlo. Los requisitos formales son también las exigidos por cada estado para estas modificaciones estatutarias. De igual forma se determina que para el caso de una fusión, intercambio de acciones, «consolidation or conversión $n^{44}$ » que tenga como resultado la terminación del estatus de una Sociedad como Benefit Corporation, también deberá ser adoptado por las mayorías exigidas legalmente. Mayorías que tal y como hemos señalado en la nota 42, normalmente son bastante reforzadas (mínimo dos tercios).

41 Esta disposición entendemos modestamente que no tiene demasiado sentido. En efecto, si lo que precisamente quieren sus promotores es que se visualice para la generalidad de los consumidores la existencia de este tipo de sociedades ¿Por qué no hacerlo constar en su denominación social?

42 Normalmente se exige una mayoría de dos tercios, por ej: en MD. (Maryland) Code Ann., Corps. \& ASS'NS Secc. 5-6C-03(b), y en Vermont STAT.ANN.tit.11. a, Secc.21.05 se exige una mayoría mínima de dos tercios; que puede ser incrementada si así se determina en los estatutos. Sin embargo en Virginia (CODE ANN. Sec. 13.1-785) se exige unanimidad de los accionistas para adoptar el acuerdo de obtener la cualificación de Benefit Corporation.

43 En este art. denominado «Definitions», se realiza una definición de los principales términos utilizados en la propuesta. Nosotros solo haremos referencia a los utilizados en el presente trabajo, según vayan surgiendo en el desarrollo del mismo.

44 Esta secc. además de regular la fusión, hace referencia a la «consolidation or conversión» que, por su escasa transcendencia práctica, y su difícil encaje en nuestra legislación, no vemos a tratar. 


\section{Sección 2. ${ }^{\text {a } . ~ O b j e t o ~ S o c i a l ~}$}

\section{Art. 201. Objetos Sociales}

La Secc. 201 (Objetos sociales), apartado (a) señala que al margen de lo dispuesto en los estatutos sociales, sobre cuál es el objeto de la Sociedad, toda Benefit Corporation, debe tener, además, como objeto el crear «a general public benefit».

General Public Benefit (que nosotros traduciremos como «interés general»), es definido en la Secc. 102 «Definitions», como «El impacto real y positivo de los negocios y operaciones de una Benefit Corporation, sobre la sociedad y el medio ambiente, entendidos como un todo; y evaluados contra las normas estandarizadas de una tercera organización independiente ${ }^{45}$ ».

En su apartado (b) se nos dice que, los estatutos sociales de una Benefit Corporation, pueden incorporar uno o más «intereses específicos» a su objeto social. Estos «intereses específicos» se añaden al del interés general señalado en el apartado (a) anterior. El MBCL añade «la determinación de un interés específico, en ningún caso limitará la obligación de la Benefit Corporation de perseguir el interés general». Además, en su apartado (c) y para cerrar el círculo se determina que "La adopción de la defensa o consecución del interés general, o de uno o varios interese específicos o particulares, según lo dispuesto en los apartados (a) y (b) anteriores, se realizará siempre en el interés de la Benefit Corporation». Si bien la última obligación vincula a los administradores de cualquier sociedad (aunque no sea Benefit Corporation), esta salvaguarda ha querido ser especialmente destacada dado el peso que en este tipo de Sociedades tiene la consecución del interés general ${ }^{46}$.

En el apartado Art. 102 (a) de la Model Benefit Corporation Legislation, también se recoge una lista meramente enunciativa de algunos objetivos que pueden ser perseguidos de forma específica por las Benefit Corporations:

1. Proveer a personas de escasos recursos materiales, o comunidades desabastecidas, con productos o servicios de los que estén necesitados.

2. Promover oportunidades económicas para individuos y comunidades más allá de la mera creación de puestos de trabajo en el desarrollo normal de los negocios.

3. Preservar el medio ambiente.

4. Luchar contra problemas de salud pública.

5. Promoción del arte, las ciencias, o el desarrollo del conocimiento.

6. Incrementar y fomentar la captación de capital para organizaciones que persigan el interés general.

45 Organización independiente, experta en el desarrollo de estándares, a nivel sectorial o global, como pueden ser B. Lab, ISO, etc

46 «White Paper. The need and rationale for the Benefit Corporation...», op. cit., p. 10. 
7. La consecución de cualquier otro beneficio en interés de la sociedad o el medio ambiente.

En la subsección (d) se permite a la Benefit Corporation el añadir, modificar o suprimir un determinado interés específico. El acuerdo deberá ser adoptado por las mayorías exigidas por la legislación de Sociedades de cada Estado.

Comentario: Algunos autores se han preguntado por qué se exige que este tipo social tenga como obligación principal, la creación del interés general, en vez de la de uno o más intereses específicos. La respuesta que nos da el libro blanco ${ }^{47}$ es que el principal objetivo de la propuesta legislativa sobre las Benefit Corporations es, la creación de beneficios para la sociedad, el medio ambiente, y los accionistas. Los empresarios sociales, y los inversores no están interesados en, por ejemplo, la reducción de la producción de residuos industriales y en cambio incrementar las emisiones de $\mathrm{CO}_{2}$; o en reducir ambas, y sin embargo obviar la creación de puestos de trabajo en comunidades con alta tasa de desempleo. Lo que interesa al legislador, es la creación de una nueva forma social que dé a los empresarios e inversores la flexibilidad y protección necesaria, para perseguir el interés general. Así mismo, se afirma ${ }^{48}$ que la exigencia de buscar este, evitará el «greenwashing» de algunas empresas que, mediante la elección de un interés demasiado específico o estrecho, podrían «olvidar» el resto de los intereses no financieros a la hora de tomar sus decisiones.

Así mismo se ha comentado que el requerimiento de perseguir «a material positive impact on society and the enviroment» es exageradamente difuso, y que el concepto de "general public benefit» demasiado amplio para ser eficaz. Para abordar esta preocupación, la $\mathrm{MBCL}$ exige que esta cuestión sea evaluada contra las normas o estándares - completos y exhaustivos - de una organización independiente y transparente en el sentido que más adelante comentaremos. El criterio de la exhaustividad, asegura que el impacto de la actuación de la Sociedad en los intereses no financieros, que los Administradores están obligados a tomar en consideración, son evaluados específicamente en el Informe del interés general anual, tal y como veremos.

Sección. III Responsabilidad

Art. 301. Estándar de conducta para los Administradores

(a) - Consideraciones de interés - En el cumplimiento de sus deberes, y considerando siempre el interés de la Benefit Corpora-

47 «White Paper...», op.cit., p. 21.

48 «White Paper...», op.cit., p. 22. 
tion, el Consejo de Administración, las diferentes comisiones del consejo, y los consejeros individuales de una Benefit Corporation:

1.1 Tendrán en consideración los efectos de cualquier acción u omisión sobre

1.1.1. Los accionistas de la Benefit Corporation.

1.1.2. Los empleados y los trabajadores de la Benefit Corporation, sus filiales, y sus proveedores.

1.1.3. El interés de los consumidores como beneficiarios del interés general o interés específico de la Benefit Corporation.

1.1.4. Factores comunitarios y sociales, incluidos los de aquellas comunidades en las que las oficinas o establecimientos de la Benefit Corporation, sus filiales o sus proveedores, se encuentran ubicadas.

1.1.5. El medioambiente local y global.

1.1.6. El interés a corto y largo plazo de la Benefit Corporation, incluyendo beneficios que podría generar la Sociedad con su estrategia a largo plazo, y la posibilidad de que estos intereses puedan ser mejor defendidos siendo una Benefit Corporation. (Este apartado quiere prevenir sobre las consecuencias que, en el negocio de la Sociedad, tendría la pérdida de la condición de Benefit Corporation).

1.1.7. Las posibilidades de la Benefit Corporation de cumplir con el interés general y, en su caso con el interés específico determinado en los estatutos sociales.

\subsection{Podrán considerar:}

1.2.1. Los intereses de algún grupo de stakeholders recogidos por la Ley de Sociedades de cada estado y que no hayan sido citados en los apartados anteriores,

1.2.2. Otros factores, o el interés de algún grupo que estimen apropiado.

1.3. No deberán dar prioridad a los intereses de una persona en particular o grupo de los citados en los epígrafes 1.1. y 1.2. anteriores, sobre los intereses de cualquier otra persona o grupo; ha menos que la Benefit Corporation haya determinado en sus estatutos su intención de dar prioridad a ciertos intereses relacionados con el cumplimiento del interés general o de un interés específico, identificado en sus artículos.

(b)... 
(c) Exoneración de responsabilidad personal. Excepto que de otra forma estuviese dispuesto en los estatutos sociales, un administrador no es personalmente responsable por los daños y perjuicios causados como consecuencia de:

c.1. Cualquier acción u omisión en el ejercicio de su cargo como administrador y teniendo en consideración lo dispuesto en el Apartado 1 (a), siempre que el administrador haya cumplido con las obligaciones impuestas por la Ley de Sociedades en general, y por esta sección en particular.

c.2. La imposibilidad de la Benefit Corporation de perseguir o crear el interés general o un interés específico.

(d) Limitación Permanente. Un Administrador no tiene obligaciones frente a una persona que sea beneficiaria del interés general o del interés específico de la Benefit Corporation; siempre que dicha obligación tenga su origen en el estatus de esa persona como beneficiario. (Es decir por el hecho de ser beneficiario del amplio campo de incluidos en un interés general público o específico, no se tiene acción contra los administradores para exigir, por ejemplo que la compañía dedique más esfuerzos a una determinada actividad o sector).

COMENTARIO. De todo lo expuesto, es interesante resaltar lo siguiente: en primer lugar que a diferencia de los Constituencies Statutes, en los que tal y como ya señalamos, el tener en cuenta los intereses de otros stakeholders distintos de los accionistas es potestativo; en la Benefit Corporation es obligatorio. La ley exige a los administradores que al tomar las decisiones de gestión del negocio, se tengan en cuenta los intereses de los stakeholders y los de los beneficiarios (tal y como se determina en el Art. 301 (a). Pero sin embargo, y tal y como veremos, estos sólo tendrán acción contra los administradores por el incumplimiento de esta obligación, sólo si expresamente están autorizados por los estatutos sociales Art. 305 (b). (Caso de no estarlo quedaría muy diluida o «aguada» la obligación contenida en el Art. 301 (a).

Art. 302 Consejero del Interés General.

(a) Esta sección precisa que las Benefit Corporations que coticen en bolsa deberán, y el consejo de cualquier otra no cotizada, podrá, nombrar a un consejero quién:

(i) Será designado como Consejero del interés general

(ii) Tendrá, además de las facultades, derechos, obligaciones e inmunidades de cualquier otro administrador de una Benefit Corporation; las facultades, derechos, obligaciones e inmunidades contenidas en este capítulo. 
(b) Elección, cese y cualificación. El Consejero del interés general, será nombrado y cesado, de acuerdo con lo dispuesto en la legislación de Sociedades. Este Consejero, será una persona física que deberá ser independiente. El Consejero del interés general, podrá ser, además el director del interés general (tal y como veremos en la Art. 304). Los estatutos podrán determinar la cualificación profesional que debe reunir este Consejero, siempre y cuando la misma no sea contraria al espíritu de este capítulo.

(c) Informe de cumplimiento anual. El Consejero del interés general deberá formular, y la Benefit Corporation deberá incluir en el informe del Interés General anual que deberá ser entregado a los accionistas, según lo dispuesto en el Art. 401 de la presente Model Legislation, la opinión del Consejero del interés público, sobre las siguientes materias:

(1) Si la Benefit Corporation ha procedido realmente de acuerdo con el interés general, y en su caso con el interés específico, durante el periodo cubierto por el informe.

(2) Si los Administradores y directivos han cumplido con lo dispuesto en los Art. 301 (a) y 303(a) respectivamente.

(3) Si, en opinión del Consejero del interés público, la Benefit Corporation o sus consejeros o directivos no actuaron o no cumplieron con lo dispuesto en los aparatdos (1) y (2) anteriores, y una descripción de la forma en la que la Benefit Corporation o sus Consejeros o directores no actuaron o cumplieron.

(d)...

(e) Exoneración de responsabilidad personal. Con independencia de si los estatutos sociales de una Benefit Corporation incluyen una disposición eliminando o limitando la responsabilidad personal ${ }^{49}$ de los Administradores, autorizada por la Ley de Sociedades, el Consejero del interés general no será personalmente responsable por un acto u omisión realizado en su cualidad de Consejero del interés público, a no ser que el acto u omisión sea declarado judicialmente como conflicto de intereses, o conducta culposa o dolosa.

49 Este tipo de disposiciones se admiten en los Estados Unidos, y son práctica habitual. Ver en Gevurtz, F., Corporation Law. Ed. West, 2. ${ }^{a}$ Edición, Minnesota, 2010, p. 329; quién comenta la modificación de la Secc. 102 del «Delaware General Corporation Law», en el sentido de limitar la responsabilidad de los Consejeros por «monetary damages». 
Comentario. Es preciso hacer notar que al margen de la exoneración de responsabilidad admitida por la mayoría de las leyes de sociedades (ver nota 49), en la que se exige que se incorpore a los estatutos para que tenga validez (es decir es opcional) en el caso de las Benefit Corporations se aplica automáticamente. Es una forma de blindar a este tipo de consejeros en su actuación, para hacer el cargo más atrayente y más independiente.

Así mismo, la opinión de este consejero sobre si la sociedad ha tenido éxito, o al menos ha tratado de perseguir el interés general público, o específico que, en su caso, haya incorporado a sus estatutos sociales, será una importante fuente de información de los accionistas al objeto de conocer hasta qué punto los administradores han cumplido con su deber para con la Benefit Corporation y sus recursos.

Art. 303. Estándares de conducta de directivos ${ }^{50}$.

(a) Regla general. Todo directivo de una Benefit Corporation deberá considerar los intereses y circunstancias descritos en el Art.301 (a), en la forma determinada en el, si:

(1) El directivo tiene capacidad de actuar discrecionalmente con respecto a una materia, y

(2) Razonablemente le parece al directivo que la materia puede tener un efecto directo en la creación, por la Benefit Corporation, del interés general o un interés específico identificado en los estatutos sociales de la Benefit Corporation.

(b)...

(c) Exoneración de responsabilidad personal. Excepto en lo dispuesto en los estatutos sociales, un directivo no será responsable personalmente de los daños y perjuicios causados por:

(1) La acción u omisión imputable al directivo en cumplimiento de sus obligaciones como directivo según lo dispuesto en la subsección (a), si el directivo las realiza de acuerdo con lo dispuesto en la Ley de Sociedades y en esta sección; o

(2) Que la Benefit Corporation no pueda perseguir o no pueda lograr la creación del interés general o del interés específico.

50 Hemos traducido como «directivos» la expresión inglesa "officer» aunque realmente sería el equivalente al personal I de alta dirección. Personal de alta dirección que, en los Estados Unidos, no tienen vínculo laboral con la compañía. 
(d) Limitación permanente. Un directivo no tiene ninguna obligación directa con ninguna persona que sea beneficiario del interés general público o del interés público específico de una Benefit Corporation; cuando la misma tenga su origen en el estatus de esa persona como beneficiario.

(e) Toma de decisiones de gestión ${ }^{51}$. Un directivo que adopta una decisión de gestión de buena fe, cumple con sus obligaciones bajo esta sección, si el directivo:

(1) No tiene intereses en el objeto de la decisión;

(2) Esta informado con respecto a la materia sobre la que se adopta la decisión, de forma que el director entienda que es suficiente bajo las circunstancias concurrentes, y

(3) Racionalmente cree que la decisión se toma en interés de la Benefit Corporation.

Comentario. Como un apoderado de la sociedad, un directivo debe seguir la instrucciones de su poderdante, pero en aquellos casos en los que el directivo no tenga instrucciones precisas de su principal, el apartado (a) exige que el directivo considere el interés de todos los stakeholders de la Benefit Corporation, en la misma forma que la exigida a los Administradores por el Art. 301.

Art. 304. Directivo del interés general.

Una Benefit Corporation puede designar a un directivo como Directivo del Interés General.

(a) Funciones. Un directivo del Interés General deberá:

(1) Tener las facultades necesarias y cumplir las obligaciones relativas al objeto de la sociedad de crear y perseguir el interés general o un interés específico, según lo dispuesto en,

(i) Los estatutos sociales,

51 Recordar que la práctica judicial norteamericana, en lo que se refiere al enjuiciamiento de decisiones empresariales adoptadas por consejeros y directivos, aplica de forma unánime la denominada «Business Judgment Rule». Según esta doctrina, los jueces no entrarán a evaluar una decisión empresarial, siempre que la misma haya sido adoptada sin perseguir fines privados y de buena fe. En el sustrato de esta doctrina esta la presunción de que la decisión ha sido adoptada con la debida diligencia. Es decir, correspondería al accionista o tercero afectado por una decisión de los directivos de una sociedad, el demostrar que estos no actuaron con la diligencia exigida. Ver en Gevurtz, F., «Corporation Law», op. cit., p. 286. 
(ii) Si no existieran disposiciones concretas en los estatutos sociales, por las instrucciones y órdenes del consejo de administración.

(2) La obligación de preparar un informe del interés general exigido por el Art. 401.

Comentario. El nombramiento de un directivo del interés general es opcional. Pero si se nombra, una de las obligaciones de ese directivo, será el preparar el Informe anual exigido por el Art. 401. Cabría preguntarse de quien es la obligación de preparar el citado informe, cuando son personas distintas el consejero y el directivo del interés general. Aunque esto no tendría demasiada lógica, es una situación que podría darse. En este caso, entendemos que debería ser el Consejero el máximo responsable, al formar parte del órgano de administración.

Art. 305. Legitimación activa.

(a) Limitación.

(1) Excepto en el caso de una acción de cumplimiento social ${ }^{52}$ [definida en el Art. 102.- Definitions], ninguna persona podrá interponer una acción, o hacer valer una reclamación contra una Benefit Corporation, sus Administradores y Directivos con respecto a:

(i) La imposibilidad de perseguir o crear el interés general o un interés específico.

(ii) El incumplimiento de cualquier obligación, deber o estándar de conducta, según lo dispuesto en este capítulo.

(2) Una Benefit Corporation no será responsable de los daños y perjuicios causados, según lo dispuesto en este capítulo, por la imposibilidad de la Benefit Corporation en perseguir o crear el interés general público o un interés público específico.

(b) Una acción de cumplimiento social, podrá ser interpuesta solo:

(1) Directamente por la Benefit Corporation; o

(2) Derivativamente (de acuerdo con las normas señaladas a estos efectos por la Ley de Sociedades) por:

52 Traducción de «Benefit Enforcement Proceeding». 
(i) Una persona, o grupo de personas que sean los propietarios finales según los datos de la compañía, de al menos, el $2 \%$ del número total de acciones de una clase o serie y que lo fueran (accionistas) en el momento del acto u omisión sobre el que se reclama,

(ii) Un Administrador;

(iii) Persona o grupo de personas que son propietarios finales, según los datos de la compañía, del $5 \%$ o más del capital de una sociedad, de la que la Benefit Corporation sea una filial, en el momento del acto $u$ omisión sobre el que se reclama.

(iv) Cualquier otra persona que expresamente autorizada en los estatutos sociales de la Benefit Corporation.

(c) Propietario Final. A los efectos de lo dispuesto en esta sección, una persona es el beneficiario final de unas acciones o de una participación en el capital, a pesar de que las acciones o las participaciónes en el capital están depositadas en uno de los llamados inversores institucionales ( $\ll$ ln a voting trust or by a nominee on behalf of the benefial owner») ${ }^{53}$.

Esta sección solo se aplica a acciones o reclamaciones que tengan su origen en lo dispuesto en este capítulo.

Comentario. La legitimación activa para el ejercicio de una acción de responsabilidad contra los Administradores y directivos de una Sociedad, por parte de una compañía (que no sea una Benefit Corporation), está limitada - en la mayoría de los estados- a la propia sociedad, o a sus accionistas. Pero esta sección amplia los legitimados, al incluir a Administradores, y a los accionistas que representen el 5\% de una sociedad filial, así como a cualquier otra persona que la propia Benefit Corporation determine mediante su expresa autorización en los estatutos sociales. Lo dispuesto en este artículo, solo será de aplicación para el ejercicio de acciones que tengan su origen en la infracción de alguna de las normas del presente capítulo.

Por lo tanto un accionista de una Benefit Corporation, podrá ejercitar la tradicional acción social de responsabilidad frente a los ad-

53 Esto es lo que la doctrina denomina «holding stock in street name», es decir cuando el registro y control directo de las acciones de la Sociedad, no lo lleva la propia sociedad, sino determinados intermediarios financieros e inversores institucionales (fondos de pensiones, públicos, privados, etc) autorizados para ello. Ver Gevurtz, F., "Corporation Law...», op. cit., p. 252, en donde literalmente dice «... beneficial owners stock held in street name, is when the corporation records show title to the shares in the name of brokers, depository companies and other nominees, rather than the actual owners». 
ministradores de la sociedad en su condición de accionista, por entender que una actuación de aquellos ha dañado sus intereses. Pero además, el mismo accionista podrá ejercitar el «benefit enforcement proceeding» (o acción específica prevista en este capítulo) contra una decisión de los administradores que no haya tenido en cuenta los intereses de otros Stakeholders o por no haberse perseguido con el suficiente "énfasis» el interés general o específico de la compañía. Así mismo, y tal y como previamente hemos anticipado, los estatutos podrán designar a una o varias personas -obviamente distintas a las que ya reconoce la ley- que tendrán la legitimación activa para el ejercicio de la «Benefit Enforcement Proceeding».

Sección IV.Transparencia.

Art. 401. Formulación del Informe del Interés General Anual54.

(a) Contenido. Una Benefit Corporation debe formular un Informe del Interés General Anual que incluya las siguientes menciones:

(1) Una descripción narrativa de

(i) Los modos en los que la Benefit Corporation ha perseguido el Interés General Público durante el año, así como el ámbito o extensión en el que el Interés General Público fue, en su caso, creado.

(ii) Ambos:

- Los modos en los que la Benefit Corporation ha perseguido el interés Público específico que los estatutos de la sociedad determinan como el objetivo a crear por la Benefit Corporation.

— el ámbito o extensión en el que el interés público específico ha sido efectivamente conseguido.

54 La traducción del término inglés "Anual Benefit Report», no nos ha sido fácil. El término finalmente elegido lo ha sido en coherencia con la traducción realizada del término «General Public Benefit» como interés general. Lo cierto es que la Ley 2/2011 de Economía sostenible, en su Art. 39 no se hace una mención expresa a este tipo de informes. Por otro lado la «Proposición de Ley sobre Responsabilidad legal de las empresas» (BOCG núm. 235-1 de 10 de Mayo de 2001) se refiere a este tipo de documentos como «Balances Sociales». Estos son definidos en el artículo 1 de la proposición como «documento que recoge los resultados analíticos sistemáticos y la evaluación de los aspectos que integran la responsabilidad social de la empresa, con o sin sujeción a una norma» Ver, para más información a este respecto Ibáñez Jiménez, J. W., Responsabilidad social de la empresa y finanzas sociales, Ed. Akal, S.A., 2004, p. 168. 
(iii) Cualquier circunstancia que haya dificultado la creación por la Benefit Corporation del interés general o del interés específico.

(iv) El procedimiento y la razón para elegir o cambiar las normas estandarizadas de una determinada organización independiente, a la hora de formular el Informe del interés general.

(2) Una evaluación global del resultado social y medioambiental de la Benefit Corporation contrastado contra los estándares de una organización independiente.

(i) Contrastado homogéneamente con cualquier aplicación de el mismo estándar en informes sociales anteriores; o

(ii) Acompañado de una explicación de las razones por las que:

- Se realiza una aplicación inconsistente o no homogénea; o

- El cambio de estándar con respecto al utilizado en el Informe anterior.

(3) El nombre del Consejero y/o Director de interés general, de existir, así como la dirección a la que poderles enviar correspondencia a cada uno de ellos.

(4) La remuneración pagada por la Benefit Corporation durante el último año a cada Consejero (o Administrador) por su condición de Consejero.

(5) La opinión del Consejero de Interés General señalada en la Secc. 302 (c).

(6) Una declaración sobre cualquier conexión55 entre la organización independiente que provee los estándares, sus

55 Ver en http://www.leginfo.ca.gov/cgi-bin/displaycode?section=corp\&group=140 01-15000\&file=14600-14604. La Legislación Californiana exige a la organización independiente que satisfaga los siguientes requerimientos: (1) Que no más de un tercio del órgano de gobierno de la organización independiente sean representantes de: (a) Una asociación de fabricantes o proveedores de servicios de un sector determinado de la actividad económica, cuyos miembros están siendo evaluados o medidos por el estándar desarrollado por la organización independiente (b) Negocios de un sector económico específico o una asociación de negocios de ese sector (c) Negocios cuya resultado, se mida contra el estándar de esa asociación.(2) Que la organización no este materialmente financiada por una asociación o negocio de los señalados en el punto (1) anterior. (3) Que sea creíble por que los estándares sean ideados y desarrollados por personas que (a) Tengan acceso al conocimiento necesario para evaluar globalmente el 
consejeros, directores, o de cualquier titular del 5\% o más de participación en el órgano de gobierno de la esta organización; y la Benefit Corporation, sus consejeros, directivos, o cualquier titular del 5\% o más de las acciones de la Benefit Corporation, incluyendo cualquier relación financiera o de gobierno que podría materialmente afectar la credibilidad del uso del estándar de la organización independiente.

(b) Cambio de Consejero del interés general. Si durante el año cubierto por un Informe, un Consejero del interés General dimitiera, o renunciara a la reelección como Consejero de interés general, o fuera cesado como Consejero de interés General, y el Consejero de interés General hubiera entregado a la Benefit Corporation cualquier correspondencia escrita relativa a las circunstancias que rodean la dimisión, la renuncia, o cese; el Informe, deberá incluir esa correspondencia como anexo.

(c) No necesidad de Auditoría. Ni el informe, ni la declaración sobre la evaluación de la Benefit Corporation en el Informe requerido por la subsección (a) (2) necesita ser auditada o certificada por una organización independiente proveedora de estándares.

Comentario: Con la información sobre la evaluación global de cumplimiento en materia social y medio ambiental a través de su confrontación con las normas (o estándares) de una organización independiente, se provee a los accionistas de una Benefit Corporation y al público en general, de una formula fácil para poder evaluar a la compañía en lo que se refiere a esos criterios. Tal y como dice el White Paper sobre las Benefit Corporation ${ }^{56}$ «De esta forma y a tra-

resultado de la actuación social y medio ambiental de la Sociedad, y (b) Utilice como criterio de desarrollo el del equilibrio entre los distintos stakeholders, incluido un periodo de información pública (para atender, en su caso sugerencias) de 30 días en el desarrollo o modificación de los estándares. (4) Transparente, porque la siguiente información, es pública: (a) Sobre el estándar (o normas estándar): (i) Los criterios utilizados a la hora de valorar el comportamiento social y medio ambiental de una empresa (ii) El peso relativo de cada uno de esos criterios. (b) Sobre el desarrollo y revisión de la norma estandarizada (i) La identidad de los Consejeros, directivos, dueños reales y órgano de gobierno de la organización que desarrolla y controla las revisiones de los estándar (ii) El proceso a través del cual las revisiones del estándar (normas) y los cambios de miembros del órgano de gobierno son realizados (iii) Una relación de los recursos financieros que sustentan a la organización, mostrada con el suficiente detalle como para detectar cualquier relación que podría, razonablemente, ser considerada como un potencial conflicto de interés. Última entrada 28 de mayo de 2013. Lo mismo que ocurre con Pennsylvania, Colorado, Illinois y Washington.

56 «White Paper...», op. cit., p. 20. 
vés de un «Due diligence» se tendrá una herramienta que facilitará una mayor inversión en las Benefit Corporations y se incrementará la lealtad de los consumidores al permitir a estos diferenciar entre «hechos» y un buen «marketing».

Art. 402 Disponibilidad del Informe del Interés General Anual.

(a) Plazos. Una Benefit Corporation deberá enviar el Informe del Interés General Anual a cada accionista:

(1) Dentro de los 120 días siguientes al cierre del ejercicio fiscal de la Benefit Corporation.

(2) Al mismo tiempo que la Benefit Corporation entregue cualquier otro informe anual a sus accionistas.

(b) Información en la página web. Una Benefit Corporation deberá publicar en su página web todos sus informes sobre el interés general. Pero tanto la remuneración de sus Consejeros, como la información financiera y de participaciones en el capital social de la compañía será omitida de los informes de esta forma publicados.

(c) Disponibilidad de copias. Si la Benefit Corporation no tiene una página web, la Benefit Corporation deberá proveer de una copia de su Informe del Interés General más reciente, sin coste, a cualquier persona que lo requiera. Pero la remuneración pagada a los Consejeros, y la información financiera y sobre participaciones en la Sociedad incluida en este Informe, serán omitidas de la copia del Informe social a enviar.

(d) Registro del Informe.

(1) A la vez que se procede al envío de las copias según lo dispuesto en la subsección (c) anterior, la Benefit Corporation deberá depositar una copia en la Secretaría de Estado ${ }^{57}$ para su registro; pero la remuneración pagada a los Consejeros y la información financiera y sobre participaciones en la compañía incluidas en el Informe del interés general, podrán ser omitidas del Informe que se deposite en la secretaria de Estado.

(2) La Secretaría de Estado cobrará una tarifa de _ Dólares por el registro del Informe Social Anual.

57 Gevurtz, F., "Corporate Law...», op. cit., p. 56, en donde se explicita que «To form the Corporation Statutes require filing the articles with a designated state agency. Typically, this is the office of the particular state's Secretary of Satate...» Es decir, el equivalente a nuestro Registro Mercantil a estos efectos. 
Lo expuesto en este apartado, es, casi en su integridad, el régimen jurídico de las Benefit Corporations tal y como se ha añadido a las leyes ó códigos de sociedades de los distintos Estados de la Unión que han incorporado a su texto este nuevo capítulo. Hemos obviado algunos apartados por entender que su examen es superfluo, o no es imprescindible para un cabal conocimiento de aquél tipo social.

\section{$\mathrm{VI}$. A modo de conclusiones}

En este último epígrafe, y dada la naturaleza descriptiva del presente trabajo, lo que realizaremos será una exposición de los puntos fuertes y debilidades de la «joven» Benefit Corporation.

En efecto, podemos señalar los siguientes meritos de este tipo social, frente a otras formas existentes en estos momentos en la legislación norteamericana: 1. La necesidad de perseguir con carácter general un interés general ó fin público, se ve acompasada con la posibilidad de adaptar sus estatutos a la finalidad de perseguir, además, un o unos fines particulares específicos. Obviamente, estos no podrán ser contradictorios con el interés general. Además con la obligación de los consejeros, de tener en cuenta el interés de otros Stakeholders, se pone fin a la inmemorial discusión en la doctrina de aquel país ${ }^{58}$ sobre si los administradores están facultados, bajo los «Constituencies Statutes» para actuar en interés de Stakeholders distintos de los accionistas. Ahora no solo pueden, sino que en virtud de un mandato legal, deben. Su incumplimiento originará responsabilidades. 2. La obligación de emitir el «anual benefit report», significa que tanto los accionistas como la sociedad en general, tendrán, al menos, una autoevaluación realizada por los administradores, sobre los problemas, logros, fracasos y éxitos obtenidos en su obligación de perseguir el interés general. En efecto, este informe, se realiza siguiendo las normas estandarizadas de una organización de reconocido prestigio, y tiene como objetivo conocer públicamente cuál ha sido la actuación de la sociedad en relación con sus responsabilidades sociales y medio ambientales. De no haberse conseguido unos objetivos razonables, los administradores podrán ser demandados por los accionistas. 3. Permite, a la opinión pública en general, y a los inversores con sensibilidad social en particular saber, de verdad, si una sociedad de esta clase está realmente comprometida

58 Fairfax., L. M., «Doing Well while doing good: Reassessing the scope of Directors fiduciary obligations in For-Profit corporations with non shareholders beneficiaries», Washigton \& Lee Law Review, vol. 59 de 2002, p. 414. 
con fines sociales y medio ambientales. Es decir que no nos encontramos ante una mera estrategia de marketing ó "green washing». 4. El ánimo de lucro de la sociedad, y la no existencia de límites al reparto de dividendos, favorecen la captación de capital. Recordemos que este ha sido siempre el argumento esgrimido como la gran desventaja de las «Social Enterprises».

Entre los puntos débiles, podemos señalar los siguientes: 1. Quizás el mayor problema al que se enfrenta la Benefit Corporation sea el que la sociedad «no haga los suficiente» en su persecución de la finalidad social ó interés general. Además existe una importante limitación en cuanto a la legitimación activa para poder exigir responsabilidades a los administradores por no perseguir con el suficiente ahínco, el interés general. Es por ello que existen autores que entienden que sería necesario un reforzamiento de esta posibilidad para poder exigir — con mayor efectividad — la finalidad social ó interés general. Por ello MUNCH ${ }^{59}$ ha propuesto una solución a este problema. Y así frente a la norma de que solo los accionistas tengan acción para exigir responsabilidades por la no consecución de estos objetivos sociales; este autor, entiende que habría que dar esta posibilidad a aquellos «stakeholders» que acrediten un «interés legítimo60».

Otra de las desventajas apuntadas es la de que los administradores se sientan más presionados por el mercado que por sus propios stakeholders, y que por lo tanto acaben primando la obtención de beneficios. Para evitar esta tentación, se propone la creación de un "Comité de Stakeholders ${ }^{61}$ » que debería controlar y asesorar a los administradores en este sentido. Además, y en relación con el Benefit Director, el mismo autor, nos dice que esta figura deberá estar potenciada al máximo. Será necesario darle más atribuciones e ir incrementando su número en proporción al tamaño de la compañía, hasta que llegue a ser una comisión del consejo, como las actualmente existentes de auditoría o retribuciones.

El sistema de información aunque ha supuesto un paso adelante incuestionable, aún es necesario perfeccionarlo, imponiendo a los admi-

59 Munch, S., «Improving the Benefit...», op. cit., p. 189.

60 Realmente no entendemos esta crítica ya que tal y como hemos visto en el Art. 305.b.2.(iv), los estatutos sociales pueden legitimar a otras personas para ejercitar la «Benefit Enforcement Proceeding». Además, pensamos que, el ampliar de esta forma tan indiscriminada la legitimación activa para el ejercicio de esta acción tendrá, sin duda, el efecto no deseado de disuadir a excelentes profesionales de aceptar el cargo de administrador de una Benefit Corporation.

61 Munch, S., «Improving the Benefit...», op. cit., p. 191. 
nistradores sanciones por la no emisión total o parcial de los informes, o por faltar a la verdad en los mismos.

Será necesario limitar el número de organizaciones contra cuyos diferentes estándares o normas se pueda confrontar el comportamiento de la sociedad a la hora de evaluar su actuación como Benefit Corporation.

El movimiento de negocios sostenibles ${ }^{62}$, la inversión socialmente responsables ${ }^{63}$ y el sector de las denominadas empresas sociales ${ }^{64}$, se está desarrollando rápidamente, pero está limitado, en los Estados Unidos, por un marco legal obsoleto que no es el adecuado para incitar la creación de Sociedades capitalistas cuya finalidad sea el ganar dinero a través de la promoción y mejora del interés general de la sociedad. La Benefit Corporation se presenta — para sus proponentes - como la más completa y flexible forma social ideada para abordar las mencionadas carencias, tratando de conjugar las necesidades de empresarios, inversores, y por extensión de los ciudadanos en general.

\section{Bibliografía}

\section{Libros}

BAINBRIDGE, S. M.: Corporate Law, Ed.Thomson/West, 2. a edición, 2009.

Benedicto XVI: Carta Encíclica "Caritas in Veritae» de 7 de julio de 2009, Ed. San Pablo, Madrid, 2009.

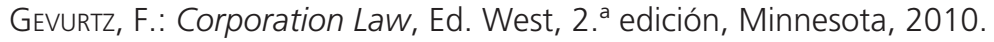

Guerra Martín, G.: El Gobierno de las Sociedades Cotizadas Estadounidenses:

Su influencia en el movimiento de reforma del Derecho Europeo, Ed. Aranzadi, Cizur Menor, 2003.

IbAÑEz Jiménez, J. W.: Responsabilidad social de la empresa y finanzas sociales, Ed. Akal, S.A., 2004.

KeAY, A.: The Corporate Objetive, Ed. Edward Elgar Publishing Limited, Massachusetts, 2011.

Romano, R.: The Genius of American Corporate Law, Ed. The AEl Press, Washigton DC, 1993.

Stout, L.: The Shareholder Value Myth, Ed. Berret-Koehlet Publishers Inc., San Francisco, 2012.

62 Ver en la U.S Chamber of Commerce http://chamber.350.org/get-your-bizinvolved/join-the-sustainable-business-movement/. Última entrada 3 de junio de 2013.

63 Ver en Global Impact Investment Network http://www.thegiin.org/cgi-bin/iowa/ investing/index.html. Última entrada 43 de junio de 2013.

64 Ver en Social Enterprises Asociation https://www.se-alliance.org/. Última entrada 3 de junio de 2013. 


\section{Revistas}

Bonini, S. MCKILlOP K, y MendOnCA, L.: «The trust gap between consumers and corporations», en The McKinsey Quarterly, vol. 10, 2007.

FAIRFAX., L. M.: «Doing Well while doing good: Reassessing the scope of Directors fiduciary obligations in For-Profit corporations with non shareholders beneficiaries», Washigton \& Lee Law Review, vol. 59, 2002

Katz, R.A. y PAge A.: "The Role of Social Enterprise», Vermont Law Review, vol. 35, 2010

Kelley, T.: "Law and Choice of Entity on the Social Enterprise Frontier», Tulane Law Review, vol. 84, 2009.

Mitchel, L.: «A theoretical and Practical Framework for Enforcing Corporate Constituency Statutes», Texas Law Review, vol. 70, 1992.

Munch, S.: «Improving the Benefit Corporation:How Traditional Governance Mechanisms can Enhance the innovative new Business Form», Northwestern Journal of Law and Social Politics, vol. 7, 2012.

Zavala ORTIZ DE LA TORRE, Iñigo. "La pugna entre el shareholder primacy model y la stakeholder theory en la doctrina y práctica anglosajona. Estado de la cuestión», Deusto Estudios Cooperativos, núm. 2, 2013.

\section{Documentos difundidos por Internet}

Benefit corporation. Net: "White paper. The need and rationale for the benefit corporation. Why it is the legal forma that best addresses the needs of social entrepreneurs, investors, and, ultimately, the public». Ver en http:// benefitcorp.net/for-attorneys/benefit-corp-white-paper.

Cone Comunications, 2010. Ver en http://www.coneinc.com/files/2007Cone SurveyReport.pdf.

Digital Media Law: http://www.dmlp.org/legal-guide/low-profit-limited-liabilitycompany.

Forbes Magazine: http://www.forbes.com/sites/annefield/2013/04/25/whatwill-be-the-first-delaware-benefit-corporation/.

Global Impact Investment Network: http://www.thegiin.org/cgi-bin/iowa/investing/index.html.

Gobierno de California: http://leginfo.legislature.ca.gov/faces/billNavClient. xhtml?bill_id=201120120SB201.

Hansonbrid: Ver en http://www.issgovernance.com/docs/2011ESGPreview..

Institutional Shareholder Service (IIs): http://www.hansonbridgett.com/ Publications/articles/2012-09-flexible-purpose.aspx

New Economy Working Group: http://www.neweconomyworkinggroup.org/ search/node/benefit\%20corporation

NoKET, N. Ver en el «The Harvard Law School Forum On Corporate Governance And Financial Regulation»: http://blogs.law.harvard.edu/corpgov/ 2012/05/13/benefit-corporations-vs-regular-corporations-a-harmfuldichotomy/ 
Social Enterprise Alliance: https://www.se-alliance.org/why\#_whatsasocialenterprise.

TAYLOR, C. en Race to the Bottom Organization: http://www.theracetothebottom.org/home/benefit-corporations-expand-but-not-without-criticism-part-1.htm.

University of Wisconsin Center for Cooperatives: STAFF PAPER N. ${ }^{\circ} 7,2008$, EN http://www.uwcc.wisc.edu/pdf/Staff\%20Papers/Staff\%20Paper\%207. pdf

U.S Chamber of Commerce: http://chamber.350.org/get-your-biz-involved/ join-the-sustainable-business-movement/. 